\title{
Investigating star formation in the young open cluster NGC $6383^{\star, \star \star}$
}

\author{
E. Paunzen, M. Netopil, and K. Zwintz
}

\author{
Institut für Astronomie der Universität Wien, Türkenschanzstr 17, 1180 Wien, Austria \\ e-mail: Ernst.Paunzen@univie.ac.at
}

Received 26 April 2006 / Accepted 18 October 2006

ABSTRACT

\begin{abstract}
Context. By studying young open clusters, the mechanisms important for star formation over several Myr can be examined. For example, accretion rate as a function of rotational velocity can be investigated. Similarly, sequential star formation triggered by massive stars with high mass-loss rates can be studied in detail.

Aims. We identified and characterized probable members of NGC 6383, as well as determined cluster parameters.

Methods. New Strömgren uvby CCD photometry, obtained by us, is presented. This new data, together with Johnson $U B V$ and 2MASS data in the NIR, was used to investigate characteristics of pre- as well as zero age main sequence cluster members.

Results. We present Strömgren uvby CCD photometry for 272 stars in the field of NGC 6383 and derive its reddening, $E(b-y)=$ 0.21 (4) mag, as well as distance, $d=1.7(3) \mathrm{kpc}$ from the Sun. Several stars with NIR excess and objects in the domain of the classical Herbig Ae/Be and T Tauri stars were detected. Two previously known variables were identified as rapidly-rotating PMS stars. The field population is clearly separated from the probable members in the color-magnitude diagram.

Conclusions. NGC 6383 is a young open cluster, with an age of less than 4 Myr, undergoing continuous star formation. True premain sequence members might be found down to absolute magnitudes of $+6 \mathrm{mag}$, with a variety of rotational velocities and stellar activities.
\end{abstract}

Key words. stars: pre-main sequence - stars: early-type - stars: formation - open clusters and associations: individual: NGC 6383

\section{Introduction}

Young open clusters offer the opportunity to investigate star formation simultaneously for a significant number of stars from high to very low mass. Processes like accretion, mass-loss and pulsation in the presence of both local and global magnetic fields can be studied (Monin et al. 2006). The pre-main sequence (PMS) phase of stellar evolution has been extensively investigated in the last decade (James et al. 2006). With the detection of magnetic fields (Wade et al. 2005) and the application of asteroseismic tools (e.g. Ripepi et al. 2006; Zwintz et al. 2006) the early phases of the stellar evolution begin to reveal their mysteries.

We investigate the southern young open cluster NGC 6383 located at $\alpha(2000.0)=17^{\mathrm{h}} 34^{\mathrm{m}} 48^{\mathrm{s}}, \quad \delta(2000.0)=-32^{\circ} 34^{\prime} 00^{\prime \prime}$; $l=355^{\circ} 690, b=0^{\circ} 041$ which has been a target of several investigations (see Zwintz et al. 2005, for a summary). This aggregate is especially interesting because it contains several variable stars and, due to a very massive binary system in its core, star formation is still ongoing (Rauw et al. 2003).

We present new Strömgren uvby photometry of several fields of NGC 6383, together with a detailed analysis of the available 2MASS data (Skrutskie et al. 2006). The color-magnitude diagram clearly shows continuous star formation over about the last 4 Myr. We have derived the cluster parameters and discovered two rapidly-rotating PMS stars. These are very interesting

$\star$ Based on observations at ESO-La Silla (Proposal 073.C-0144).

$\star \star$ Data table is only available in electronic form at the CDS via anonymous ftp to cdsarc.u-strasbg.fr $(130.79 .128 .5)$ or via http://cdsweb.u-strasbg.fr/cgi-bin/qcat?]/A+A/462/157 objects for further observations. An analysis of the NIR data shows that PMS members are present to spectral types of M0 or even cooler. Some members show a NIR excess and are located in the photometric domain of Herbig Ae/Be and classical T Tauri stars. Based on all available information from our observations and the literature, a list of bona-fide members of NGC 6383 was compiled.

\section{Observations and reduction}

The observations were performed during the night of 13./14.06.2004 at the 3.6 m telescope (ESO-La Silla), with the EFOSC2 (fast modus) and the Loral/Lesser $2048 \times 2048$ pixel (1 pixel = 0.'157) CCD, which yields a 5' field-of-view using a standard Strömgren uvby filter set (Observer: M. Netopil).

As NGC 6383 is believed to be rather extended, about $20^{\prime}$, we have chosen five overlapping fields in the cluster area to cover the most interesting objects. In total, 33 frames in each filter were observed with integration times ranging from 5 to $300 \mathrm{~s}$.

The bias-subtraction, dark-correction, flat-fielding and pointspread-function fitting were carried out within standard IRAF V2.12.2 routines.

No offsets in instrumental magnitudes between the different overlapping fields were detected, within the photometric and transformation error limits.

The accurate uvby photometry of IC 4651 published by Meibom (2000) was used to transform our instrumental magnitudes to standard ones. We have observed IC 4651 at different airmasses resulting in 25 individual frames. After correcting all frames for the airmass, the following standard relations were 


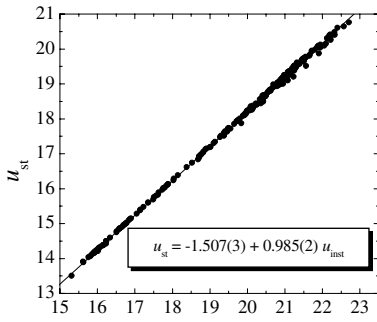

$u_{\text {inst }}$

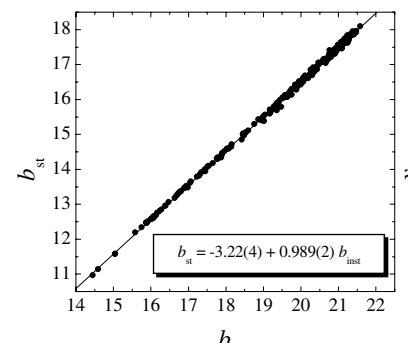

$b_{\text {ins }}$

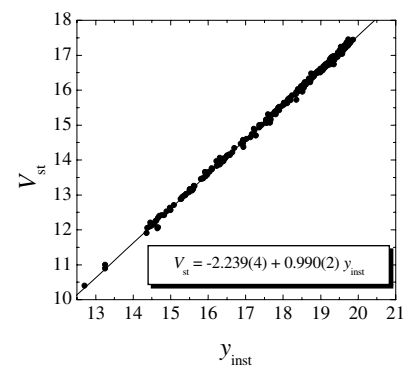

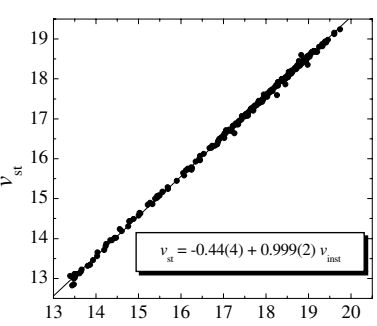

$v_{\text {inst }}$

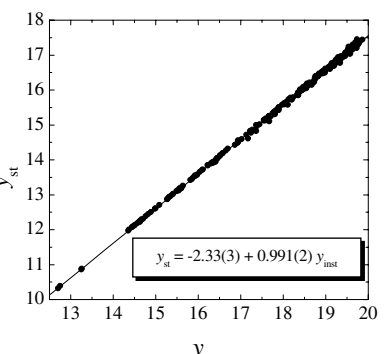

$y_{\text {inst }}$
Fig. 1. The standard relations of our instrumental magnitudes (suffixes "inst") to the highly accurate $u v b y$ photometry of IC 4651 published by Meibom (2000).

derived (Fig. 1). The errors in the final digits of the corresponding quantity are given in parentheses.

$$
\begin{aligned}
u_{\text {st }} & =-1.507(3)+0.985(2) u_{\text {inst }} \\
v_{\text {st }} & =-0.441(3)+1.000(2) v_{\text {inst }} \\
b_{\text {st }} & =-3.220(3)+0.986(2) b_{\text {inst }} \\
y_{\text {st }} & =-2.262(3)+0.991(2) y_{\text {inst }} \\
V_{\text {st }} & =-2.239(4)+0.990(2) y_{\text {inst }} .
\end{aligned}
$$

Those relations were applied to the instrumental magnitudes of the stars in the five fields of NGC 6383.

For a final test, we compared our uvby photometry with data from Eggen (1978) for six stars in both our sample and his. He used a narrower $v$ filter which only effects $m_{1}$ and $c_{1}$. The values agree very well within the error limits.

In total, we observed 272 stars, of which 105 could not be measured in Strömgren $u$ because of their relative faintness and the limited quantum efficiency of the instrument.

The table with all the data for individual cluster stars, as well as nonmembers, is available in electronic form at the CDS or upon request from the first author. The table includes our internal numbers, the WEBDA and 2MASS identifiers, J2000.0 coordinates, the $X$ and $Y$ coordinates within our frames, the observed $(b-y), m_{1}$ and $c_{1}$ values with their corresponding errors, $V$ magnitudes, the $(B-V)$ colors from the literature and the number of observations, respectively.

\section{Results}

Throughout this paper we have adopted the numbering system from WEBDA (http: //www . univie.ac.at/webda), only in some cases we also list the numbers according to FitzGerald et al. (1978, F\#) and Zwintz et al. (2005, Z\#).

NGC 6383, which is associated with a large H II region, was targeted for several investigations in the past. They have been summarized by Zwintz et al. (2005) in some detail. The basic parameters of this cluster, from different sources, are: $E(B-V)=0.31(3) \mathrm{mag}, 1.4<d<2.5 \mathrm{kpc}$ and $\log t<4$ Myr. Its apparent diameter is still a matter of debate. FitzGerald et al. (1978) concluded on the basis of star counts (see Fig. 1 therein) that only a core radius of $2^{\prime}$ should be considered, whereas Zwintz et al. (2005) give a value of $10^{\prime}$, which is based on Lyngå (1987). Kharchenko et al. (2005) list a core and cluster radius of 4.8, and $15^{\prime}$, respectively. Their age estimate of $5 \mathrm{Myr}$ is compatible with the literature whereas their distance of $985 \mathrm{pc}$ seems too low.

Figure 2 shows the observed five fields with the location of members and non-members. The sizes (by area) of the symbols are inversely proportional to the $V$-magnitudes. Inspecting the area surrounding NGC 6383 in the Digitized Sky Survey (DSS), one can see several empty areas, probably caused by dark clouds. Dobashi et al. (2005) have also listed that region in their Atlas and Catalog of Dark Clouds, reporting a cloud and clumps extending over $0.27^{\circ}$ in this area. On the basis of this fact, a density profile using, for example, the extensive 2MASS data will evoke erratic results for the cluster diameter. However, a cluster radius of $10^{\prime}$ to $15^{\prime}$ seems to be within the realm of possibility, since the confirmed members are distributed over the whole observed field of $\sim 15^{\prime}$ (Fig. 2). From the comparison of Fig. 2 with the DSS images, we conclude that we have covered only $60 \%$ of the complete cluster area, introducing an additional bias. Only further photometric observations can help establishing the diameter of NGC 6383.

All members of NGC 6383 which are later than a spectral type of A0 are still in their PMS phase. Rauw et al. (2003) studied the cluster in X-rays using data from the XMM-Newton satellite. They detected several X-ray sources, which were interpreted as signs of young T Tauri stars. FitzGerald et al. (1978) concluded that the central bright $(V=5.7 \mathrm{mag})$ spectroscopic binary $(\mathrm{O} 7 \mathrm{~V}+\mathrm{O} 7 \mathrm{~V})$ system, HD 159176 (WEBDA No. 1), triggered star formation within its surroundings. They derive an age of 2.8(5) Myr for it.

No estimation of the cluster metallicity exists in the literature. There are neither spectroscopic abundance investigations nor a photometric determination available. The latter is caused by the fact that only stars hotter than $\mathrm{A} 0$ are on the zero age main sequence and no giant or supergiant is a member of NGC 6383. Photometric metallicity calibrations are only available for giants (Hilker 2000) or stars later than A0 (Karaali et al. 2005). A query in WEBDA reveals that there are no other open clusters in the galactic vicinity of NGC 6383 with a known metallicity. The galactic distance, $R_{\mathrm{GC}}$, of NGC 6383 is about 5.0 to $6.0 \mathrm{kpc}$ depending on its true distance from the Sun $\left(R_{0}=8.0 \mathrm{kpc}\right)$. Taking a mean abundance gradient of $-0.04 \mathrm{dex} \mathrm{kpc}^{-1}$ (Cunha \& Daflon 2005) for the Milky Way, an upper limit for the metallicity of -0.12 dex is evident. Such a small difference from the solar value does not alter the isochrone significantly (Girardi et al. 2002). However, it has to be emphasized that the "intrinsic" range of metallicities for a constant $R_{\mathrm{GC}}$ is about $\pm 0.25 \mathrm{dex}$ (Chen et al. 2003), but data for open clusters in the direction to the galactic center is still very rare. 


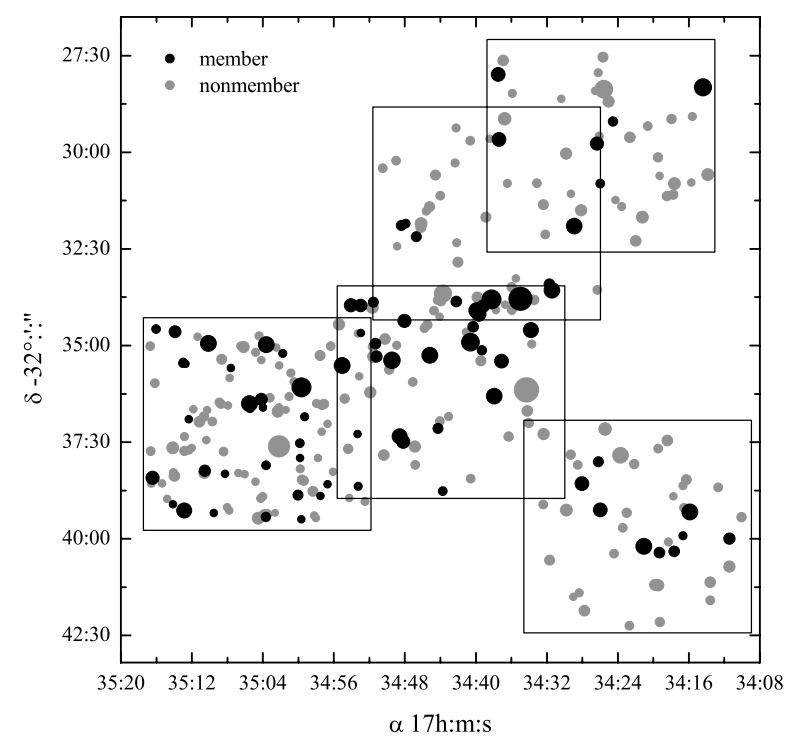

Fig. 2. The five observed fields in NGC 6383. The sizes (by area) of the symbols are inversely proportional to the $V$-magnitudes.

\subsection{Optical properties}

For the further analysis, we have checked the intrinsic consistency of the available Johnson $U B V$ photometry included in WEBDA. In total, twelve independent data sources are available. The only systematic offset was found for the $(B-V)$ data by Zwintz et al. (2005). One has to keep in mind that the cited photometry was not obtained for an astrophysical analysis of cluster properties, but to detect pulsating members. Hence, the reduction was tuned for high relative photometric accuracy on time scales of hours. Not surprisingly, a re-reduction of the data in conformity with Johnson standards arrives at slightly different values. Using the method described in Mermilliod \& Paunzen (2003), a second-order polynomial fit to the deviations (middle panel of Fig. 3) provides an intrinsically more accurate transformation to the Johnson system. We have not included these data in our further analysis.

The PMS evolutionary tracks by Siess et al. (2000) were fitted to the cluster's color-magnitude diagram, and used to determine the cluster's distance and reddening. These tracks are listed for luminosity and effective temperature, respectively. The transformation of luminosity into absolute magnitude is straightforward. More of a problem is the transformation of effective temperature into Strömgren $(b-y)$ because several calibrations are available. First of all, the empirical ZAMS listed by Philip \& Egret (1980) was used to convert the ends of the PMS tracks into the $(b-y)$ and $M_{V}$ plane. We found that the calibration by Hauck \& Künzli (1996, Table 1) satisfies the color-magnitude diagram best. The results by Clem et al. (2004) confirm our choice. The final transformation is given as

$$
\begin{aligned}
& \Theta_{\mathrm{eff}}=+0.564(8)+2.961(23)(b-y)_{0} \quad(b-y)_{0}<0 \\
& \Theta_{\mathrm{eff}}=+0.564(9)+0.822(3)(b-y)_{0} \quad(b-y)_{0}>0 \\
& \Theta_{\mathrm{eff}}=\frac{5040 \mathrm{~K}}{T_{\mathrm{eff}}}
\end{aligned}
$$

including a small shift of +0.02 mag compared to the original equations to match the standard ZAMS line by Philip \& Egret (1980).

Figure 4 shows the color-magnitude diagram with an adopted reddening of $E(b-y)=0.21 \mathrm{mag}$ and a distance
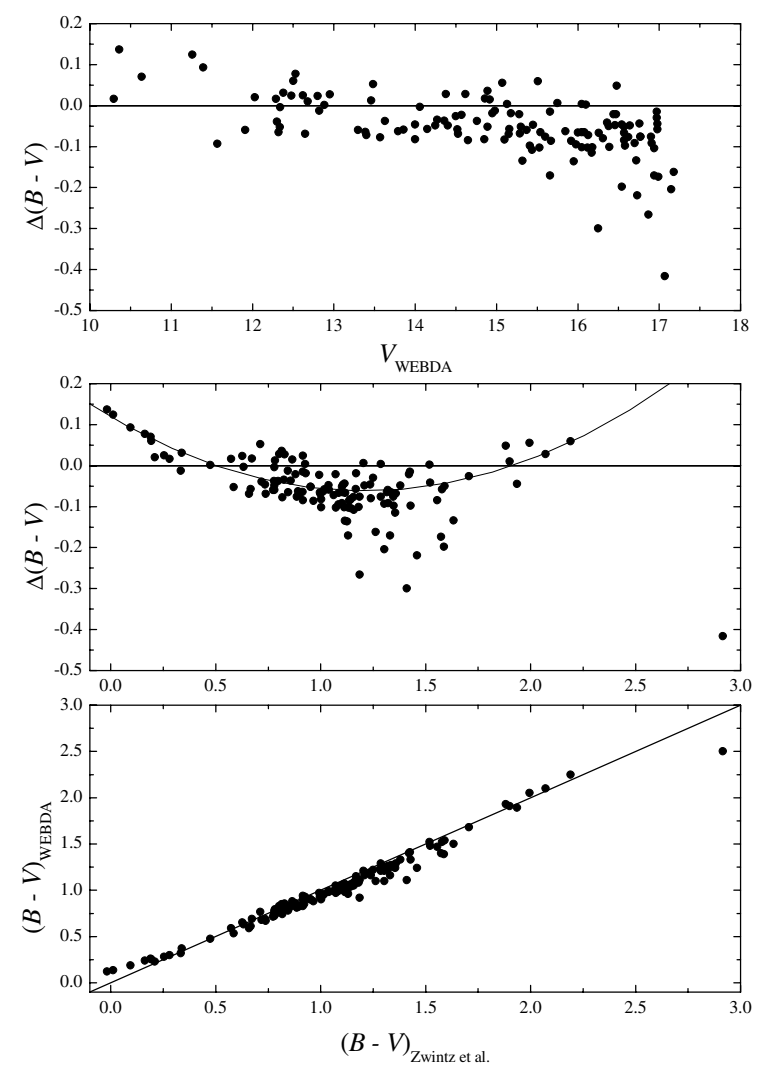

Fig. 3. Johnson $B V$ photometry by Zwintz et al. (2005) in comparison with the mean photometric values from WEBDA. The mean $\Delta(B-$ $V)=(B-V)_{W}-(B-V)_{Z}$ for the intermediate region $(0.5<(B-V)<1.5)$ is about $0.07 \mathrm{mag}$, whereas for hotter and cooler stars there is a systematic trend which can be described by a second-order polynomial.

modulus $V-M_{V}$ of $12.0 \mathrm{mag}$. This corresponds to $E(B-$ $V)=0.29 \mathrm{mag}$ and a distance of about $1.7 \mathrm{kpc}$. The objects marked with filled circles are members according to the literature, asterisks represent the possible PMS members taken from Table 1. The stars with absolute magnitudes brighter than +1 mag are identified non-members taken from the literature (e.g. FitzGerald et al. 1978). Figure 5 shows the $(U-B)_{0}$ versus $(B-V)_{0}$ for cluster members, with the standard line from Girardi et al. (2002), computed for $E(B-V)=0.29$ mag and an error limit of $\pm 0.05 \mathrm{mag}$. This error band represents the main sequence up to $(B-V)_{0}=+0.1$ mag very well. Cooler objects are still in their PMS phase and can have a peculiar behavior in this diagram. The $\pm 0.05 \mathrm{mag}$ error corresponds to $\Delta E(b-y)=0.036$ and $\Delta A_{V}=0.155 \mathrm{mag}$. In Fig. 5, two outliers are noticeable, the objects No. 17 and 55. These two stars show no other abnormal behavior, which prevents any reliable conclusion about their nature. The star No. 55 is variable according to Zwintz et al. (2005) with an amplitude of about $25 \mathrm{mmag}$, which is too small to explain the apparent shift.

The observed color-magnitude diagram (Fig. 4) shows very interesting features. First of all, there is a lack of objects with absolute magnitudes between +0.5 and +2.0 mag. The only exception is object No. 264, for which a large reddening in the NIR is evident (Fig. 6). This gap seems to be the borderline between the members of NGC 6383 and the field population. The members in the PMS phase lie all within the isochrones between 1 to $4 \mathrm{Myr}$ down to $M_{V}=2.5 \mathrm{mag}$ (F0). These are PMS A-type objects mixed together with Herbig Ae/Be stars 


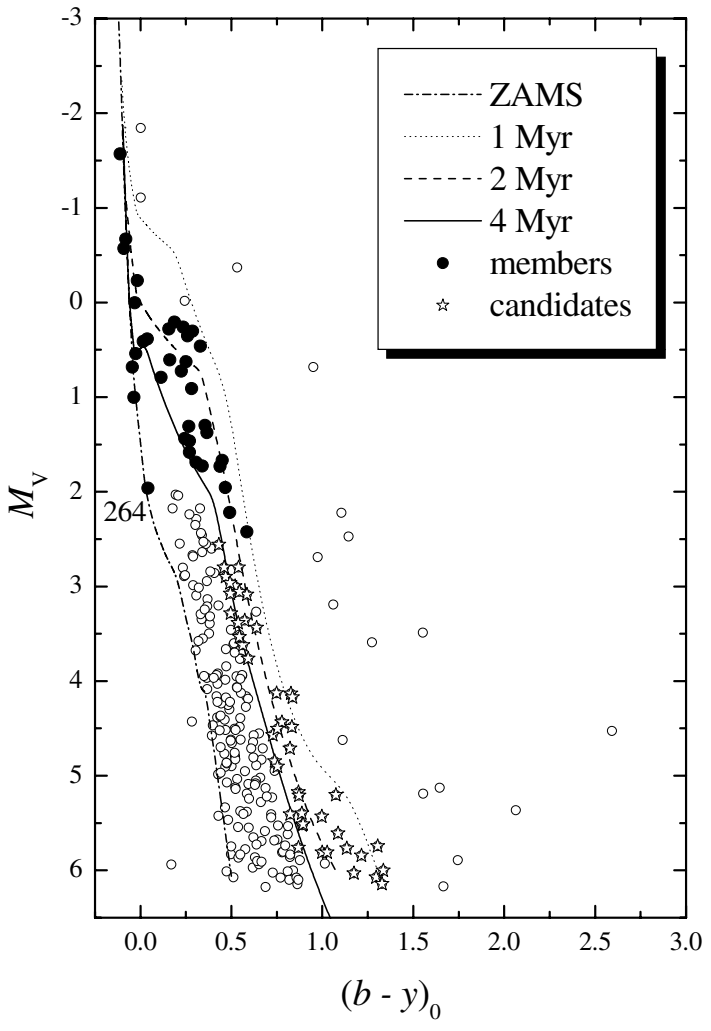

Fig. 4. Color-magnitude diagram assuming $E(b-y)=0.21 \mathrm{mag}$ and $V-M_{V}=12.0 \mathrm{mag}$. The zero age main sequence is taken from Philip $\&$ Egret (1980) whereas the pre-main sequence tracks are from Siess et al. (2000). Non-members brighter than +1 mag were selected from the literature (e.g. FitzGerald et al. 1978).

(Hernandez et al. 2005). The classical T Tauri stars have spectral types later than G0 (Meyer et al. 1997) which corresponds to $M_{V} \approx 4.3 \mathrm{mag}$.

The estimation of distance error is difficult because neither a turn-off point nor red giants are present in NGC 6383. That is the reason why widely different distance estimations (1.4 to $2.5 \mathrm{kpc}$ which corresponds to $\Delta M_{V} \approx 1.1 \mathrm{mag}$ for a constant reddening) for this aggregate can be found in the literature. From Fig. 4 we are able to make a rough estimation of the distance error by inspecting the location of members with respect to the ZAMS and PMS tracks. Taking into account the error in reddening for both parameters we can still fit all features, within observational errors, with a shift of $\pm 0.4 \mathrm{mag}$. The final cluster parameters and their errors are: $E(B-V)=0.29(5) \mathrm{mag}$ and $d=1.7(3) \mathrm{kpc}$.

\subsection{Near-Infrared properties}

The 2MASS data base (Skrutskie et al. 2006) with Near-Infrared measurements (NIR) was queried for appropriate entries in a radius of $10^{\prime}$ around the cluster's center, covering the five observed fields. The coordinates of these objects were then transformed into a rectangular $(X, Y)$ frame and compared with the star positions within our images. We have only included objects with 2MASS $(J H K)$ measurements within two arc seconds of the optical source and with unambiguous identifications. This resulted in 262 positive detections.

We have used the 2MASS data to search for signs of NIR excess and circumstellar dust. The results of the method described by Suchkov et al. (2002), analyzing the $\left(V-K_{\mathrm{S}}\right)$ versus $(b-y)_{0}$ diagram, are in total agreement with the much more accurate conclusions from the $(J-H)_{0}$ versus $\left(H-K_{\mathrm{S}}\right)_{0}$ diagram, shown in Fig. 6, for all bona-fide and possible members.

The standard lines for the main sequence included in Fig. 6 are from Bessell \& Brett (1988) with the reddening vectors according to Rieke \& Lebofsky (1985). The differences between the 2MASS photometric system and the one from Bessell \& Brett (1988) are only marginal, in the range of a few hundredths of a magnitude (Carpenter 2001, Appendix A). The locus of the classical T Tauri stars is from Meyer et al. (1997), whereas the region of Herbig Ae/Be and emission line F-type objects is taken from Hernandez et al. (2005). Notice that the weakemission T Tauri stars can not be separated from main sequence objects (Meyer et al. 1997). We find five objects with a significant NIR-excess:

- Stars with known NIR excess: Nos. 20, 27 (F4) and 264 (F6) were targets of the investigation by van den Ancker et al. (2000). The amount of excess nicely correlates with the deviation from the standard relation for normal type stars within our diagram (see their Fig. 2).

- New detections: Nos. 13 and 32, both show a moderate excess, which makes them interesting targets for a spectroscopic investigation.

The star No. 264 is highly reddened, probably caused by circumstellar material. There are also a few probable members which show similar but less extreme behavior.

For the final step, we have investigated all cool stars located inside the PMS tracks (Fig. 4) for which 2MASS measurements are available. Fourteen of them are placed in the domain of Herbig $\mathrm{Ae} / \mathrm{Be}$ and $\mathrm{T}$ Tauri stars, with thirty additional objects located in the region of the standard relation (Fig. 6). Ten objects have available $(U-B)$ measurements (Fig. 5), placing them well on the standard relation given the cluster's reddening. Table 1 lists the 44 objects which are good candidate PMS members of NGC 6383, selected on the basis of the above mentioned criteria. Further spectroscopic and kinematic data are required to establish their nature and membership.

\subsection{Two rapidly rotating PMS stars}

We would like to comment on two very interesting stars, No. 379 (Z64) and 382 (Z71), published by Zwintz et al. (2005), for which our new photometry adds important information. Both stars fall well within the PMS domain in the NIR (Fig. 6) and can be regarded as members of NGC 6383. This is supported by the reddening-free $\left[m_{1}\right]$ values of 0.414 and $0.525 \mathrm{mag}$ respectively, which are typical for stars later than K (Strömgren 1966). The hypothesis that both are highly reddened B-type stars, as formulated by Zwintz et al. (2005), can therefore be excluded. The angular velocities of both objects are about 16 radians $\mathrm{d}^{-1}$ or approximately 30 to $40 \%$ of their break-up velocity, if we assume that the found periods are due to rotation. Such high values are not typical for PMS stars (Herbst et al. 2002) but have been observed in similar objects (Strassmeier et al. 2005). Rapidlyrotating PMS stars are most important for understanding the accretion process combined with the effects of stellar magnetic fields (Eisner et al. 2005).

\section{Conclusions}

We have investigated the young open cluster NGC 6383 with several photometric systems, presenting new Strömgren uvby CCD photometry for 272 stars. From this data we derive a reddening of $E(b-y)=0.21(4) \mathrm{mag}$ and a distance of 
Table 1. A list of the 44 probable members of NGC 6383 that are still in their PMS phase. These objects were selected on the basis of several photometric diagrams. The column "Flag" denotes stars that are in the PMS region in the NIR diagram (Fig. 6).

\begin{tabular}{rcccc|rcccc}
\hline \hline No(our) & No(WEBDA) & $(b-y)_{0}$ & $M_{V}$ & Flag & No(our) & No(WEBDA) & $(b-y)_{0}$ & $M_{V}$ & Flag \\
\hline 8 & 348 & 0.823 & 4.71 & $*$ & 163 & 538 & 0.997 & 5.43 & \\
13 & 222 & 1.134 & 5.77 & & 164 & 335 & 1.027 & 5.81 & \\
21 & & 0.868 & 5.18 & $*$ & 169 & & 0.868 & 5.76 & \\
23 & & 0.872 & 5.21 & $*$ & 178 & 596 & 0.563 & 3.62 & \\
26 & & 0.890 & 5.52 & $*$ & 179 & 607 & 0.520 & 3.00 & \\
28 & 206 & 0.999 & 5.81 & & 180 & 611 & 0.539 & 2.80 & \\
36 & 444 & 0.461 & 2.80 & & 191 & 378 & 0.545 & 3.54 & \\
44 & & 1.087 & 5.61 & $*$ & 193 & 379 & 0.738 & 4.85 & $*$ \\
55 & & 1.216 & 5.84 & & 197 & 382 & 0.574 & 3.36 & $*$ \\
61 & 343 & 0.894 & 5.52 & $*$ & 206 & 251 & 0.537 & 3.39 & \\
73 & 199 & 1.074 & 5.20 & $*$ & 211 & 535 & 0.728 & 4.57 & \\
86 & & 1.296 & 6.07 & & 217 & 388 & 0.494 & 3.08 & $*$ \\
87 & & 0.828 & 4.13 & & 226 & 604 & 0.585 & 3.09 & \\
90 & 543 & 0.778 & 4.44 & & 231 & 609 & 0.748 & 4.13 & \\
106 & 337 & 0.824 & 5.41 & $*$ & 273 & 128 & 0.592 & 3.76 & \\
116 & 541 & 0.639 & 3.44 & $*$ & 277 & 503 & 0.544 & 3.06 & \\
118 & 546 & 0.832 & 4.49 & $*$ & 297 & 313 & 0.494 & 2.97 & \\
119 & & 1.335 & 6.00 & $*$ & 301 & 571 & 0.753 & 4.50 & \\
120 & & 1.175 & 6.03 & $*$ & 307 & 566 & 0.837 & 4.17 & \\
126 & 134 & 0.888 & 5.40 & $*$ & 308 & 312 & 0.497 & 3.29 & \\
136 & 539 & 1.305 & 5.75 & & 312 & 310 & 0.753 & 4.91 & \\
143 & & 1.328 & 6.14 & & 328 & 309 & 0.432 & 2.56 & \\
\hline
\end{tabular}

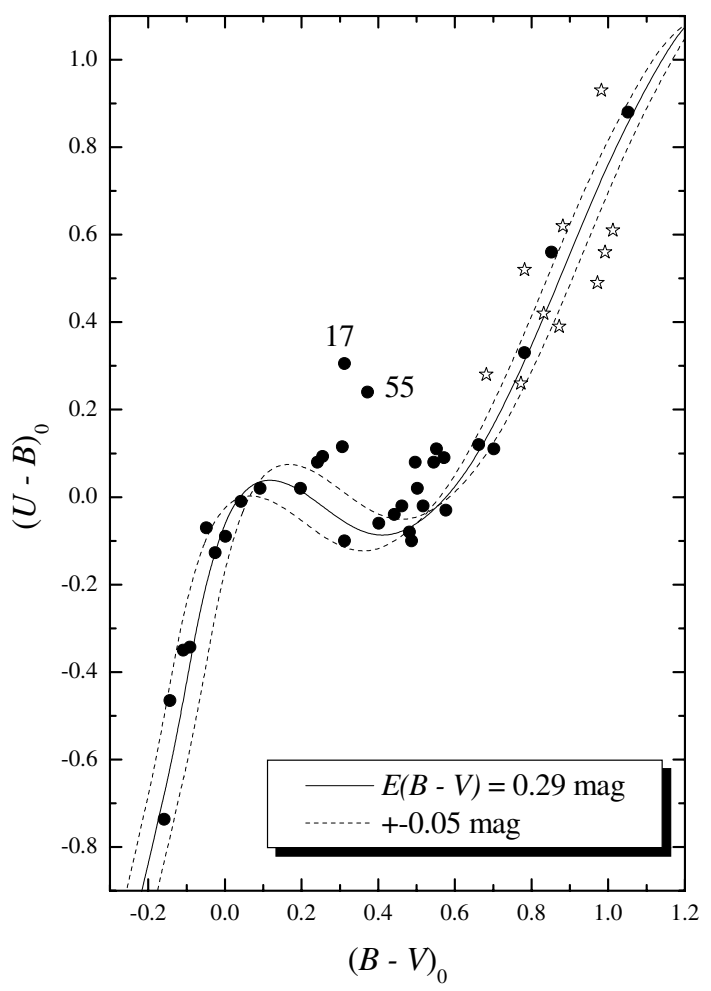

Fig. 5. $(U-B)_{0}$ versus $(B-V)_{0}$ diagram for the members (filled circles) and possible members (asterisks). The standard line is taken from Girardi et al. (2002) computed for $E(B-V)=0.29$ mag and an error limit of $\pm 0.05 \mathrm{mag}$. The only significant outliers are the stars No. 17 and 55 (known as variable).

$d=1.7(3) \mathrm{kpc}$. An upper age limit of $4 \mathrm{Myr}$ was determined for NGC 6383. Neither a turn-off point nor red giants have been detected.

In the $M_{V}$ versus $(b-y)_{0}$ color-magnitude diagram, 44 probable PMS members could be traced from A to very cool M-type

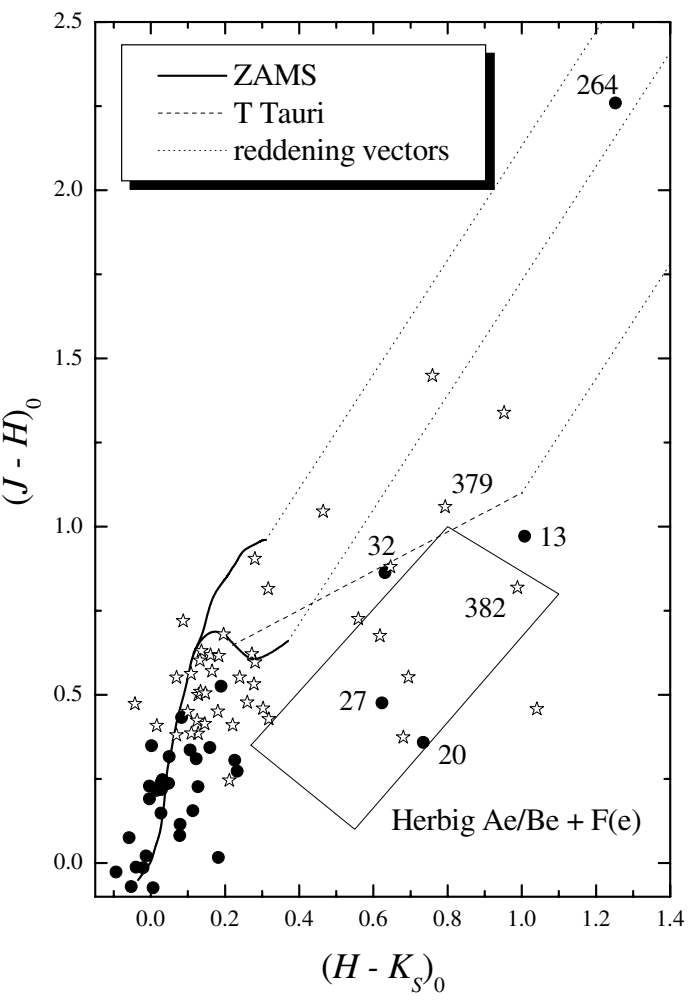

Fig. 6. $(J-H)_{0}$ versus $\left(H-K_{\mathrm{S}}\right)_{0}$ diagram. The standard lines for the main sequence are from Bessell \& Brett (1988), with the reddening vectors (dotted lines) according to Rieke \& Lebofsky (1985). The locus of the classical T Tauri stars (dashed line) is from Meyer et al. (1997), whereas the region of Herbig $\mathrm{Ae} / \mathrm{Be}$ and emission line F-type objects is taken from Hernandez et al. (2005).

objects. At least 14 of them are in the NIR (2MASS) domain of classical T Tauri stars. Appropriate isochrones clearly show that members are present from 1 to $4 \mathrm{Myr}$ with a distinct separation from the field population at absolute magnitudes between +0.5 and $+2.0 \mathrm{mag}$. 
Five stars with a large NIR excess are unambiguously identified in a $(J-H)_{0}$ versus $\left(H-K_{\mathrm{S}}\right)_{0}$ diagram.

We also report the identification of two rapidly-rotating PMS stars with angular velocities of approximately 30 to $40 \%$ of their break-up velocity. Such objects are still very rare and most important for out understanding of the early stages of stellar evolution, in the presence of accretion and local magnetic fields.

Acknowledgements. We would like to dedicate this paper to Hartmut Holweger who died during its preparation. This research was performed within the projects $P 17580$ and $P 17920$ of the Austrian Fonds zur Förderung der wissenschaftlichen Forschung (FwF). K. Zwintz acknowledges the support by the project P14984 of the FwF. Use was made of the WEBDA database, operated at the University of Vienna, the NASA's Astrophysics Data System and data products from the Two Micron All Sky Survey, which is a joint project of the University of Massachusetts and the Infrared Processing and Analysis Center/California Institute of Technology, funded by the National Aeronautics and Space Administration and the National Science Foundation. These observations have been funded by the Optical Infrared Coordination network (OPTICON), a major international collaboration supported by the Research Infrastructures Programme of the European Commission's Sixth Framework Programme.

\section{References}

Bessell, M. S., \& Brett, M. 1988, PASP, 100, 1134

Carpenter, J. M. 2001, AJ, 121, 2851

Chen, L., Hou, J. L., \& Wang, J. J. 2003, AJ, 125, 1397

Clem, J. L., VandenBerg, D. A., Grundahl, F., \& Bell, R. A. 2004, AJ, 127, 1227

Cunha, K., \& Daflon, S. 2005, in Cosmic Abundances as Records of Stellar Evolution and Nucleosynthesis in honor of David L. Lambert, ed. Th. G. Barnes, \& F. N. Bash, ASP Conf. Ser., 336, 103

Dobashi, K., Uehara, H., Kandori, R., et al. 2005, PASJ, 57, 1
Eggen, O. J. 1978, AJ, 83, 288

Eisner, J. A., Hillenbrand, L. A., White, R. J., Akeson, R. L., \& Sargent, A. I. 2005, ApJ, 623, 952

FitzGerald, M. P., Jackson, P. D., Luiken, M., Grayzeck, E. J., \& Moffat, A. F. J. 1978, MNRAS, 182, 607

Girardi, L., Bertelli, G., Bressan, A., et al. 2002, A\&A, 391, 195

Hauck, B., \& Künzli, M. 1996, Baltic Astronomy, 5, 303

Herbst, W., Bailer-Jones, C. A. L., Mundt, R., Meisenheimer, K., \& Wackermann, R. 2002, A\&A, 396, 513

Hernandez, J., Calvet, N., Hartmann, L., et al. 2005, AJ, 129, 856

Hilker, M. 2000, A\&A, 355, 994

James, D. J., Melo, C., Santos, N. C., \& Bouvier, J. 2006, A\&A, 446, 971

Karaali, S., Bilir, S., \& Tuncel, S. 2005, PASA, 22, 24

Kharchenko, N. V., Piskunov, A. E., Röser, S., Schilbach, E., \& Scholz, R.-D. 2005, A\&A, 438, 1163

Lyngå, G. 1987, Catalogue of Open Cluster Data, 5th ed. (Strasbourg: CDS)

Meibom, S. 2000, A\&A, 361, 929

Meyer, M. R., Calvet, N., \& Hillenbrand, L. A. 1997, AJ, 114, 288

Mermilliod, J.-C., \& Paunzen, E. 2003, A\&A, 410, 511

Monin, J.-L., Menard, F., \& Peretto, N. 2006, A\&A, 446, 201

Philip, A. D., \& Egret, D. 1980, A\&AS, 40, 199

Rauw, G., De Becker, M., Gosset, E., Pittard, J. M., \& Stevens, I. R. 2003, A\&A, 407, 925

Ripepi, V., Bernabei, S., Marconi, M., et al. 2006, A\&A, 449, 335

Rieke, G. H., \& Lebofsky, M. J. 1985, ApJ, 228, 618

Siess, L., Dufour, E., \& Forestini, M. 2000, A\&A, 358, 593

Skrutskie, M. F., Cutri, R. M., Stiening, R., et al. 2006, AJ, 131, 1163

Strassmeier, K. G., Rice, J. B., Ritter, A., et al. 2005, A\&A, 440, 1105

Strömgren, B. 1966, ARA\&A, 4, 433

Suchkov, A. A., Schultz, A. B., \& Lisse, C. M. 2002, ApJ, 570, L29

van den Ancker, M. E., The, P. S., \& de Winter, D. 2000, A\&A, 362, 580

Wade, G. A., Drouin, D., Bagnulo, S., et al. 2005, A\&A, 442, L31

Zwintz, K., Marconi, M., Reegen, P., \& Weiss, W. W. 2005, MNRAS, 357, 345

Zwintz, K., Guenther, D. B., \& Weiss, W. W. 2006, ApJ, in press 